\title{
Erratum to: A diffusion tensor imaging and neuropsychological study of prospective memory impairment in South African HIV positive individuals
}

\author{
Jacqueline Hoare • Jenny Westgarth-Taylor • \\ Jean-Paul Fouche • Bruce Spottiswoode • Robert Paul • \\ Kevin Thomas • Dan Stein • John Joska
}

Published online: 22 August 2013

(C) Springer Science+Business Media New York 2013

Erratum to: Metab Brain Dis (2012) 27:289-297

DOI 10.1007/s11011-012-9311-0

The original version of this article unfortunately contained mistakes. The author's given name and family name were interchanged. The author names are now corrected in this article.

\footnotetext{
The online version of the original article can be found at http://dx.doi.org/ 10.1007/s11011-012-9311-0.

J.-P. Fouche

MRC Research Unit on Anxiety Disorders, Department of

Psychiatry, Stellenbosch University, Stellenbosch, South Africa

J.-P. Fouche $\cdot$ B. Spottiswoode

Fisan Building Faculty of Health Sciences, Tygerberg Hospital,

Cape Town, South Africa 7500

J. Hoare $(\bowtie) \cdot J$. Westgarth-Taylor $\cdot$ D. Stein $\cdot$ J. Joska

Department of Psychiatry and Mental Health, University of Cape

Town, Anzio Road Observatory, 7925 Cape Town, South Africa

e-mail: hoare.jax@googlemail.com

R. Paul

Department of Psychology and Behavioural Neuroscience,

University of Missouri, St. Louis, One University Blvd Stadler 412,

St. Louis, MO 63121, USA

K. Thomas

ACSENT Laboratory Department of Psychology, University of

Cape Town, Cape Town, South Africa 7925

B. Spottiswoode

MRC/UCT Medical Imaging Research Unit, Department of Human Biology, University of Cape Town, Cape Town, South Africa 7925

B. Spottiswoode

Department of Radiological Sciences and Oncology, Stellenbosch

University, Stellenbosch, South Africa
} 